\title{
Narrativa
}

\section{Il ruolo e il valore del denaro in Kamikaze d'Occidente di Tiziano Scarpa}

Laura Rinaldi

\section{(2) OpenEdition}

1 Journals

Edizione digitale

URL: https://journals.openedition.org/narrativa/332

DOI: $10.4000 /$ narrativa.332

ISSN: 2804-1224

\section{Editore}

Presses universitaires de Paris Nanterre

\section{Edizione cartacea}

Data di pubblicazione: 1 décembre 2020

Paginazione: 153-164

ISBN: 978-2-84016-381-7

ISSN: $1166-3243$

Notizia bibliografica digitale

Laura Rinaldi, «ll ruolo e il valore del denaro in Kamikaze d'Occidente di Tiziano Scarpa», Narrativa [Online], 42 | 2020, online dal 01 décembre 2021, consultato il 08 décembre 2021. URL: http:// journals.openedition.org/narrativa/332 ; DOI: https://doi.org/10.4000/narrativa.332

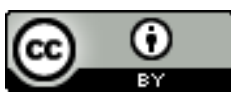

Narrativa est mise à disposition selon les termes de la Licence Creative Commons Attribution 4.0 International. 


\title{
Il ruolo e il valore del denaro in Kamikaze d'Occidente di Tiziano Scarpa
}

\begin{abstract}
RIASSUNTO
L'articolo si propone di analizzare il romanzo Kamikaze d'Occidente di Tiziano Scarpa rispetto alla centralità che assume al suo interno il medium del denaro. L'opera, a cavallo tra gli stilemi postmoderni e il nuovo millennio, soffre ancora le influenze di tendenze letterarie passate, come quella dei Cannibali; al contempo, l'aspetto più propriamente economico contribuisce a proiettarla in avanti rendendolo adatto a essere inserito in un dibattito sulla letteratura italiana iper-contemporanea.
\end{abstract}

\section{RÉSUMÉ}

Cet article se propose d'analyser le roman Kamikaze d'Occidente de Tiziano Scarpa par rapport à la centralité jouée par l'argent dans la narration. Cette œuvre, qui oscille entre l'esthétique du postmoderne et celle du nouveau millénaire, reste soumise aux influences de tendances littéraires revolues, comme celles des Cannibali; en même temps, sa dimension économique est telle qu'elle finit par intégrer le débat sur la littérature italienne hyper-contemporaine.

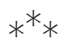

Il romanzo Kamikaze d'Occidente di Tiziano Scarpa connette l'esperienza letteraria di fine xx secolo e le linee innovatrici dei primi anni Zero. Pubblicato per la prima volta nel 2003 e poi nel 2019', lega insieme un "mondo nuovo" - forgiato dai grandi eventi di inizio millennio - e forme letterarie che hanno

1. La prima edizione del 2003 è pubblicata da Rizzoli, mentre la seconda del 2019 è per minimum fax. In questo articolo si fa riferimento alla seconda, nella quale vengono aggiunte e al contempo eliminate alcune cartelle e nella quale viene inserita una postfazione dello stesso autore in cui egli si esprime sulla genesi e la struttura del romanzo stesso. 
segnato la letteratura dagli anni Sessanta in poi. Vale allora la pena soffermarsi sulla natura della narrazione e sulle influenze che vengono esercitate su di essa per comprendere quale sia il nucleo centrale del racconto e per tentare un'analisi delle istanze che lo motivano.

Sin dalla pubblicazione di Occbi sulla graticola ${ }^{2}$, Scarpa entra di diritto a far parte dei Cannibali e con loro condivide tematiche e stilemi che si caratterizzavano - se non nella totalità almeno in parte - per una vicinanza al postmoderno. Ma è un postmoderno, questo, lontano da quello letterario e molto più vicino invece allo stile iperviolento e citazionistico cinematografico ${ }^{4}$. L'attenzione quasi ossessiva - per la sessualità ${ }^{5}$, alcune ibridazioni con la cultura pop e la mescolanza tra codici linguistici alti e bassi permangono anche in Kamikaze d'Occidente a distanza di quasi dieci anni, in un contesto storico-letterario ormai mutato. Scarpa si trova insomma ad accostare all'estetica fine anni Novanta strutture che trascendono la mera fiction ${ }^{6}$. Si è ancora troppo vicini all'esperienza

2. SCARPA Tiziano, Occhi sulla graticola. Breve saggio sulla penultima storia d'amore vissuta dalla donna alla quale desidererei unirmi in duraturo vincolo affettivo, Torino, Einaudi, 1996.

3. Scarpa non è presente nella antologia di riferimento Gioventù cannibale del 1996 che dà il la a tutta la corrente, ma pubblica - nello stesso anno - il già citato Occhi sulla graticola. Il '96 segna indubbiamente l'inizio per il fenomeno letterario con la pubblicazione anche di Woobinda di Aldo Nove, Fango di Niccolò Ammanniti, e Stanotte e per sempre di Daniele Luttazzi.

4. Le radici del postmoderno possono essere rintracciate tra il decennio della contestazione e il primo grande sviluppo economico postguerra: "the postmodern emerged in what I call the long sixities, extending from the late fifties to the early seventies; from the heyday of the Beat movement and the rise of popular youth culture to Watergate" (Dekoven Marianne, Utopia Limited, Durham, Duke University Press, 2004, p. 4). Il decennio degli anni Sessanta, anticipato e posticipato a piacimento, è, dunque, il riferimento per la nascita della letteratura metanarrativa di Barth, Pynchon e Gaddis; è però diversa la datazione per quanto riguarda il cinema, che invece trova la sua nascita con gli stilemi del movimento solo a partire dai tardi anni Ottanta e, poi con maggior forza, negli anni Novanta. La corrente letteraria italiana "pulp" o "cannibale" prende forma da questa seconda espressione e si lega a tutta una diramazione di moduli rappresentativi che poggiano la loro cifra stilistica, oltre che sulla cultura pop e le nuove tecnologie, su film quali Pulp fiction (da cui prende in prestito il nome) e The Big Lebowski.

5. Cfr. per esempio il brano seguente: "le si innesca un'inculata meditativa, come sempre nelle donne che lo prendono nel culo di rado. In questi casi, trasferiscono tutta l'anima sull'orlo del forellino, centuplicando l'attenzione sensoriale e intellettuale sullo sfintere. Soppesano il dolore e il significato di ciò che stanno facendo. La stranezza, l'intensità della sensazione fisica acuisce la riflessione, invece di sovrastarla. Le rende ipercontemplative. L'inculata è la sorgente del filosofare", Scarpa Tiziano, Kamikaze d'Occidente, Roma, minimum fax, 2019, p. 7.

6. È una tendenza questa che definisce gli anni del nuovo secolo e ne segna la definitiva rottura con le correnti letterarie precedenti. Una lucida disanima si può trovare in Ipermodernità di Donnarumma dove si traccia una linea di continuità innovatrice 
di quegli autori definiti "ex cannibali o ex pulp" e, più nello specifico, permane la difficoltà di slegarsi definitivamente da quel tipo di narrazione. In altre parole Scarpa cede ancora al richiamo di una letteratura che poggia le basi sull'ironia e la parodia e, per lui, "la corruzione postmoderna occidentale rimane una culla troppo comoda per essere abbandonata" .

Eppure, anche alla luce di questa prolungata vicinanza al postmoderno e alla resistenza che il romanzo mantiene verso una rappresentazione troppo mimetica della realtà, vi sono degli elementi di originalità che rendono Kamikaze d'Occidente un emblema di come la letteratura contemporanea possa ancora muovere verso una descrizione del mondo anche senza ricercare nuove forme di realismo. L'elemento di peculiarità del romanzo risiede nell'attenzione posta alla società italiana di inizio del nuovo secolo e, più nello specifico, nella condizione economica del paese. Seguendo ancora l'analisi di Donnarumma, l'intreccio può essere delineato in questo modo:

Diario-romanzo che uno scrittore gigolo alter ego dell'autore tiene su commissione del Ministero cinese per la Finzione, e dal cui esito dipende se la potenza orientale risparmierà o distruggerà l'Europa e gli Stati Uniti. Da un lato l'esibizione pornografica e narcisistica è protratta con tale convinzione e insistenza da rendere flebile persino una taccia di manierismo; dall'altro i fatti di Genova e l'11 settembre tornano come eventi simbolici che incrinano il compiacimento insoddisfatto e onanistico del narratore ${ }^{8}$.

Si è di fronte alla cronaca stralunata di uno scrittore che assiste a eventi sconvolgenti della storia mondiale attraverso la lente della propria esistenza privata, della vita quotidiana che si alterna tra incontri sessuali retribuiti e la carriera di scrittore e intellettuale in un'Italia immobile anche di fronte ai mutamenti della Storia. Per quanto siano interessanti tutti gli aspetti del romanzo nella loro natura singola, è necessario analizzarli alla luce di un elemento che assume il ruolo di motore della narrazione: il denaro. Con questa premessa è possibile avanzare l'idea che esista in quest'opera una prospettiva di carattere teorico che

tra esempi che si distaccano completamente dal canone postmoderno, come Gomorra di Roberto Saviano, fino a esempi più o meno in linea con le nuove tendenze come Mi chiamo Roberta, ho 40 anni, guadagno 250 euro al mese... di Aldo Nove e lo stesso Kamikaze d'Occidente, che però risulta il più fallimentare nell'intento di distaccarsi dalla postmodernità.

7. Donnarumma Raffaele, Ipermodernità. Dove va la narrativa contemporanea, Bologna, il Mulino, 2014, p. 83.

8. Ibid., p. 82. 
innova rispetto alla corrente pulp, quest'ultima ormai tacciata di aver intrapreso un percorso discendente?

Una volta dimostrato che esiste una correlazione tra postmoderno e pulp, allora sarà possibile individuare quali siano gli elementi che ne possano permettere, in qualche modo, il superamento. In Postmodernist Fiction McHale traccia la linea di demarcazione tra modernismo e narrativa postmoderna individuando il cambio di dominante interno; se il primo si muove su una ricerca interna vincolata alla conoscenza e quindi di natura epistemologica, la seconda ha invece un carattere che tende a questionare e scardinare le conoscenze sull'essere e la realtà e, perciò, ha una natura ontologica ${ }^{10}$. L'opera di Scarpa muove un passo in avanti rispetto a questo sistema binario scardinandolo; l'attenzione non è infatti posta né sulla natura della conoscenza né tantomeno sull'interpretazione del mondo, quanto invece sul dato materiale, su un elemento concreto (il denaro) che, come un demiurgo, plasma l'esistere contemporaneo. Così, il denaro si erge a protagonista assoluto della narrazione vincolando ogni personaggio a relazionarsi e definirsi in base a esso; diviene elemento di scambio quindi mezzo - ma anche scopo finale, orizzonte di senso. Ciò rappresenta uno scarto decisivo verso il materiale, verso l'esistente nella sua più assoluta concretezza. Se la letteratura postmoderna rigettava le grandi metanarrazioni, Scarpa in Kamikaze d'Occidente rinnega nella loro totalità elementi svincolati dall'esperienza quotidiana. Neanche gli atti terroristici che si susseguono per l'intero arco narrativo servono a distogliere da ciò che davvero conta e cioè dal proprio tornaconto economico personale. La contrapposizione insita nello stesso titolo evidenzia fin da subito una contraddizione interna: il significato messianico del kamikaze diventa secondario rispetto a un'esperienza che tende solo apparentemente a un valore salvifico, e che appare invece mossa unicamente dalla promessa di guadagno. La prospettiva del protagonista che - per scelta del committente cinese - dovrebbe essere quella della compilazione di un'opera

9. A questo proposito un intervento chiave è il saggio di Gianni Turchetta, "I cannibali non mordono più", nel quale è centrale la disamina di un fenomeno letterario che sembrerebbe essersi ripiegato su se stesso perdendo la "cattiveria" che lo distingueva: "è possibile fare i cannibali di professione senza imborghesirsi troppo, e senza incagliarsi nello stereotipo dello scrittore?" (TURCHETTA Gianni, "I cannibali non mordono più", in Giovani scrittori e personaggi giovani, a cura di Vittorio Spinazzola, Tirature '05, Milano, Fondazione Mondadori-il Saggiatore, 2005, p. 10).

10. McHale Brian, Postmodernist Fiction, Londra-New York, Routledge, 1987. McHale aggancia la sua analisi partendo da un saggio di Jakobson nel quale viene appunto definita la dominante come la componente chiave di ogni opera d'arte. La corrente postmoderna viene definita da una dominante ontologica, seguendo McHale, per la sua strutturale separazione dal mondo dell'esperienza in favore di un "otherness of the fictional world" (p. 27). 
che descriva e, con ciò stesso, tenti di salvare la società occidentale cede invece il passo al perseguimento esclusivo del compenso promesso al termine della prestazione. Cambia quindi la motivazione alla base del racconto: non è più centrale la conoscenza e la comprensione del mondo esterno, bensì, il singolo dato concreto che se ne ricava, in questo caso: il denaro.

"Dei kamikaze dice che credono di essersi procurati un posto in paradiso 'dove gli eroi si scopano le Urì'. Io lo faccio dal vivo, per lavoro. E mi tocca scriverlo per salvare L'Occidente"'11. Il lavoro diviene quindi tema portante del testo commissionato al protagonista perdendo al contempo ogni possibile valenza di riscatto. La ricompensa oltremondana dei kamikaze è qui, infatti, di natura fortemente immanente; in alternativa a un paradiso eterno vi è un mondo finito il cui piacere ultimo non è quello della carne ma il benessere economico. Il narratore attraversa i mesi dalla primavera all'inverno del 2001 senza essere realmente influenzato da eventi al di fuori delle proprie attività remunerative, sia come scrittore retribuito per il suo servizio al governo cinese sia per il suo lavoro di natura sessuale con le clienti. Il denaro è il vero agente a cui egli risponde, il leitmotiv che muove ogni sua azione:

Nel mio libro, il sesso è in primo piano, con una ricorsività di performance di arte contemporanea. Gli episodi erotici vengono scambiati con una dimensione ipertemporale: i soldi. Da una parte il tempo, dall'altra il denaro. Da una parte gli incontri sessuali, dall'altra il loro compenso, la traducibilità di ogni esperienza in tariffa. Il valore dell'esperienza e il suo prezzo. L'entusiasmo e il suo costo. Il pathos e il suo importo. L'imponderabilità e il suo conteggio ${ }^{12}$.

Il denaro, prima ancora del suo valore di scambio, è dunque il nucleo della storia; un nucleo che dirige le sorti di tutti i personaggi in gioco ${ }^{13}$. Tutto all'interno del romanzo viene capitalizzato e filtrato dalla dinamica della domanda-offerta. Non solo si assiste alla mercificazione della voce narrante, che si prostituisce, ma della sua stessa immagine di intellettuale (con le clienti) e del suo ruolo di scrittore (con il funzionario cinese). L'azione principale si fonda, quindi, da un lato sulla mercificazione della persona, sulla reificazione del soggetto, e dall'altro sulla compravendita di beni relazionali. E si tratta di

11. Scarpa Tiziano, Kamikaze d'Occidente, cit., p. 266.

12. Ibid., pp. 354-355.

13. Al fine di avere una visione più ampia della rappresentazione e interpretazione del denaro nella letteratura contemporanea e del suo ruolo quale simbolo e simulacro si rimanda a Letteratura e denaro: ideologie, metafore, rappresentazioni: atti del XLI Convegno interuniversitario, Volume 29 di Quaderni del Circolo filologico-linguistico padovano, Bressanone, 11-14 luglio 2013. 
una finanziarizzazione tanto estrema della vita e della società da portare con sé immancabilmente un senso di sconfitta: "if the reign of finance in the circle of capital accumulation is always a sign of Autumn, we seem finally to have arrived at the onset of Winter" ${ }^{\prime 14}$. Lo stesso alter ego di Scarpa, al limite della sua autoconsapevolezza, percependosi ormai come strumento di lavoro, rinnega persino le sue pulsioni e i suoi bisogni per ottenere un guadagno:

"Non mi masturberò mai più", ho pensato, "non farò più esercizi spirituali, né con la pornografia né con la fantasia, se questo è lo strepitoso risultato di quattro giorni di astinenza. Vale davvero la pena di non diluire il piacere, se a letto posso rendere così bene professionalmente, e anche guadagnare così tanto" ${ }^{\prime 15}$.

Assunto che il denaro è posto con forza all'interno del romanzo in quanto cardine e vincolo per il diramarsi delle vicende e per la costruzione dei rapporti interpersonali, è necessario definire quale sia il valore di questo strumento e quale ruolo ingaggi nella società descritta da Scarpa. Sin dalle prime pagine, tra le battute che il narratore scambia con il funzionario cinese, l'intento dell'opera è chiaro: narrare le vicende della vita di un tipo - più che mai in senso lukacsiano - per poi presentarle alla popolazione cinese al fine di rendere ben visibile le devianze e le falle del sistema capitalista occidentale. Abbiamo, quindi, subito le coordinate di un mondo, Europeo e Nordamericano in particolare, immerso in un contesto globalizzato e liberista. Questa commissione comunica tuttavia, benché implicitamente, l'idea che questo tipo di sistema economico sia fallimentare e insostenibile e sia la causa di una deriva sociale ${ }^{16}$. Unica via di fuga per la salvezza sarebbe l'individuazione - attraverso le pagine di questo diario sui generis - di una scintilla di passione ${ }^{17}$ che ancora potrebbe soggiacere in un Occidente alla deriva. L'attenzione esasperata all'attività sessuale del protagonista

14. Clover Joshua, "Autumn of the System: Poetry and Financial Capital, in Journal of Narrative Theory, Vol. 41, n. 1 (Primavera 2011), p. 34.

15. Scarpa Tiziano, Kamikaze d'Occidente, cit., p. 280.

16. Nella sua metanarratività il romanzo assume consapevolezza della sua stessa posizione sul mercato, essendo un diario scritto su commissione lo stesso romanzo riflette anche sul suo stesso valore economico. Questa tendenza non è nuova alla letteratura dal postmoderno in poi e in un recente studio è stata analizzata approfonditamente: Crosthwaite Paul nel suo The Market Logics Of Contemporary Fiction individua un approccio che - dai classici della metafiction degli anni Sessanta ai primi anni Zero - definisce come market metafiction.

17. "Un barlume di speranza, una promessa di miglioramento, altrimenti per quale motivo dovremmo prenderci il disturbo di venire a casa vostra a governarvi con rettitudine e saggezza, quando faremmo prima a cancellarvi dalla faccia della terra?", Scarpa Tiziano, Kamikaze d'Occidente, cit., p. 37. 
con le sue clienti, che attraversa l'intero arco narrativo, non lascia spazio a quella scintilla però, che il committente aveva definito come unica chiave di lettura per una possibile redenzione.

Le spinte contrastanti del processo di globalizzazione creano dunque due ordini di problemi morali, quelli che riguardano il rapporto individuo-altri e quelli che riguardano il rapporto dell'individuo con se stesso; il primo ordine di problemi sfocia in un pluralismo che può anche diventare relativizzazione delle norme morali, il secondo in una pluralizzazione delle scelte che rischia la frammentazione dell'identità ${ }^{18}$.

L'alter-ego di Scarpa soffre appieno degli strascichi della crisi di fine secolo e non riesce a liberarsi appieno da una situazione di stallo e di distorsione della realtà. Trova risposta alle discrasie della propria vita solo attraverso la commercializzazione del proprio corpo. Mentre la carriera di scrittore non decolla, la politica e la società italiana stentano ad avere un ruolo regolatore concreto, e la regolazione dei rapporti personali passa solo attraverso il medium del denaro. Quest'ultimo si configura come strumento di strutturazione sociale, mezzo con il quale regolare la propria posizione all'interno dello schema di interazione con altri individui e all'interno della società stessa che viene suddivisa nel romanzo proprio in base all'attitudine che si ha verso la moneta. Da un punto di vista sociale, si forma una struttura piramidale che coinvolge clienti-narratore-funzionario e ne definisce il valore sociale in riferimento al loro rapporto con la moneta. Se alla base di questa piramide troviamo le clienti, che si trovano spesso nella posizione di negare la retribuzione allo scrittore/gigolò, all'apice di essa troviamo invece il committente cinese, talmente ricco da evitare persino di parlare (volgarmente) di cifre. All'interno di questa struttura di potere ben codificata e assoluta, il denaro assume un ruolo antagonistico rispetto ai sentimenti, alle emozioni e alla profondità delle relazioni umane. Sono proprio le clienti, spinte da sentimenti, ad accorgersene e a rifiutare i soldi del compenso: "e adesso che insisto per pagarti con il mio amore tu pensi solo ai soldi che ti devo?"19.

Uno dei testi chiave per capire il ruolo che assume il denaro nella società contemporanea è Filosofia del denaro di Simmel2 ${ }^{20}$ Va formandosi una

18. Bovone Laura, "Globalizzazione e frammentazione: i paradossi della cultura postmoderna", in Studi di Sociologia, Anno 33, Fasc. 1, gennaio-marzo 1995, p. 14.

19. Scarpa Tiziano, Kamikaze d'Occidente, cit., p. 122.

20. Simmel Georg, Filosofia del denaro [1900], Cavalli Alessandro, Perucchi Lucio (a cura di), Torino, UTET, 1984. 
predisposizione, all'interno di dei rapporti tra le persone, che prevede ragionamenti di tipo matematico e impersonale, e secondo Simmel, è il predominio delle funzioni intellettuali rispetto a quelle affettive a creare relazioni affette da una sordità emotiva. Il denaro contribuisce a creare rapporti e scambi in cui le dinamiche legate al sentimento, quando non eliminate, sono sottomesse a logiche economiche, a una razionalità strumentale e dunque, di conseguenza, a comportamenti individualisti ed egoisti, sia nei rapporti sociali (al lavoro ad esempio) sia nei rapporti più intimi e familiari. L'approccio di Simmel poggia le basi su una concezione del denaro fortemente vincolata all'analisi della teoria sociologica del valore ${ }^{21}$. In divergenza con la filosofia neokantiana, che radica la legittimità del valore su un principio trascendentale, e da Marx e Ricardo che individuano un valore antropologico nel lavoro, Simmel caratterizza il valore esclusivamente in rapporto a dinamiche della realtà sociale. Il denaro e il suo valore sono vincolati allo sviluppo della società moderna in cui si crea un cortocircuito che porta all'impersonalità dei rapporti umani. Il denaro diviene così all'inizio del xx secolo uno strumento mimetico della società e dell'economia, capace di descrivere una realtà dove non solo gli oggetti sono merci, ma anche $\mathrm{i}$ valori e i rapporti umani vengono valutati in base a calcoli matematici. L'uomo stesso si fa meccanismo e ingranaggio del processo economico e ne diventa uno strumento fondamentale e perciò calcolabile, deriva questa che poggia le basi dall'inizio della modernità e che segna un punto di non ritorno nel finire del xix secolo.

Se, però, il sociologo rintraccia questo scambio economico come base del principio di alienazione dell'individuo causato dalla divisione del lavoro, il protagonista del romanzo di Scarpa si trova in una condizione analoga ma molto radicalizzata. Non è più il nuovo ruolo dell'uomo nella sua condizione moderna a creare estraniamento, ma l'ormai comune impossibilità come individuo di definirsi all'interno del mondo, non tanto per un'estrema segmentazione del lavoro, quanto piuttosto per una difficoltà nel conseguimento del proprio percorso secondo volontà individuali e spinte personali.

La programmazione sul futuro delle carriere professionali, economiche, sociali e personali e delle intersezioni rischia però di avere dell'utopistico nell'attuale

21. L'attenzione per questo aspetto è legata alla letteratura critica che si è concentrata sull'opera di Simmel a partire dagli anni Ottanta in poi, un testo cardine è sicuramente il saggio CANTÒ MiLÀ Natàlia, A Sociological Theory of Value: Georg Simmel's Sociological Relationism, Bielefeld, Transcript, 2005, disponible anche online: https:// www.academia.edu/1479030/A_Sociological_Theory_of_Value_Georg_Simmels_ Sociological_Relationism. 
quadro di destrutturazione, o meglio di individuazione e individualizzazione, in cui è diventata più complessa la progettazione coerente dei propri percorsi di vita. Gli incroci tra carriere dell'individuo, e tra carriere, cambiamenti istituzionali e congiunture economico-finanziarie, concorrono dunque a definire l'attuale passaggio del rischio dal sistema all'individuo ${ }^{22}$.

È chiaro che le valutazioni di Simmel nascono in un contesto che non è quello attuale, e che fanno riferimento a dinamiche e a scambi i che non appartengono più alla nostra realtà; eppure rimane evidente la connessione che nasce dal rapporto che si crea tra soggetto e denaro e la distorsione interiore, antropologica, che questa dinamica tende a provocare.

In Kamikaze d'Occidente il denaro ha un ruolo tanto pervasivo da assumere le fattezze di agente regolatore e distruttore allo stesso tempo, e ciò è tanto più vero se osserviamo ciò che esso produce sulle relazioni umane. Questa prospettiva, che riflette sulle interazioni sociali alla luce del potere del capitale (potremmo dire: della politica alla luce dell'economia), non è nuova in letteratura ma assume un ruolo rilevante a partire dalla seconda metà del secolo scorso. In particolare, l'idea che il capitalismo e il tardo capitalismo scardinino la struttura relazionale (la famiglia, i rapporti intimi etc.) torna con forza nella narrativa occidentale a partire dagli anni Settanta del xx secolo.

Molta narrativa statunitense (per lo più postmoderna) muove da premesse simili: la svalutazione dei rapporti umani è dovuta al demone del guadagno e alla rincorsa al denaro, e il capitale (inteso come una sorta di Moloch distruttivo) è uno dei suoi topoi preferiti. Il testo più rappresentativo è probabilmente $J R$ di William Gaddis $^{23}$ : il mondo della finanza e della speculazione che viene rappresentato provoca infatti non solo la mercificazione dei rapporti umani ma anche la loro completa sostituzione con il denaro stesso. Il protagonista di $J R$ procede meccanicamente alla distruzione della famiglia - allegorica solo in parte - a favore di una famiglia di compagnie, finendo lui stesso per divenire strumento

22. Moiso Valentina, "I fenomeni finanziari nella letteratura sociologica contemporanea: l'emergenza di nuove prospettive", in Stato e mercato, 92 (2), agosto 2011, p. 329.

23. Gaddis William, JR, Alfred A. Knopf, New York, 1975. JR è qui citato come emblema, nonostante sia un romanzo lontano dall'esperienza sia cronologica che spaziale di Scarpa. La lista delle opere americane che si allineano a questa rappresentazione del denaro dal secondo dopoguerra sarebbero tante, tra le altre Money: $A$ Suicide Note di Martin Amis e Cosmopolis di Don DeLillo meriterebbero una menzione particolare. 
del capitale ${ }^{24}$. Come ha scritto Ralph Clare, "JR, a natural spokesperson for capital, is not so much speaking for capital as capital is speaking through him"25. Lo scarto di Scarpa è però dettato dalla situazione economica italiana. Se nelle rappresentazioni di Gaddis domina il principio di accumulazione della ricchezza nel romanzo di Scarpa la prospettiva è al ribasso perché la ricchezza serve alla sussistenza. Da una parte c'è l'alta finanza e le corporazioni, dall'altra c'è la precarietà, la quale spinge gli individui ad azioni impulsive e talvolta gratuite.

La voce narrante di Kamikaze d'Occidente - che pur rivela un autocompiacimento egotico - diverge da quella postmoderna: il legame con il denaro non è infatti dovuto a un principio superiore e per lo più inconoscibile ma a una spinta vitale (tutt'al più vitalistica) concreta e materiale:

Se riesco a procurarmi clienti a sufficienza, è perché faccio lo scrittore. L'attività di scrittore, infatti, mi conferisce uno status erotico presso una fascia limitata, ma per me economicamente sufficiente, di fruitrici dei miei servizi sessuali e sentimentali. E d'altro lato, se posso permettermi di fare lo scrittore senza essere costretto ad andarmi a guadagnare uno stipendio in una ditta, è grazie a questa attività interpersonale remunerata. È un circolo virtuoso ${ }^{26}$.

La centralità del capitale è vincolata alla precarietà dell'esistenza, dell'intellettuale e del lavoratore contemporaneo. Contraltare del denaro, lungo l'asse narrativo, è quindi la precarietà che affonda le radici in ogni aspetto della vita dello scrittore e del mondo che lo circonda. Una precarietà che all'interno del romanzo assume diverse sfaccettature, principalmente la precarietà relativa ad avvenimenti storici e sociali che si susseguono nell'arco dei mesi del 2001, ancora una precarietà nell'ambito affettivo-romantico che si risolve nell'impossibilità di accedere ad un sentimento che non sia di sola natura carnale, e infine una precarietà che coinvolge l'ambito puramente lavorativo, eternamente avvolto dall'instabilità.

Il romanzo, che si apre con la volontà di trovare un "barlume di speranza" all'interno della società capitalista, registra un fallimento su più fronti. La

24. Il romanzo, estremamente sperimentale nella forma, tratta l'ascesa di un ragazzo - JR - nel mondo delle speculazioni finanziarie fino a rendersi demiurgo della creazione di un reticolato di imprese che acquisiscono la conformazione di un vero e proprio nucleo familiare, sostitutivo a quello di carattere umano. L'unica motivazione che l'undicenne sente è quella derivante dagli affari, impermeabile a qualsiasi slancio di tipo relazionale e affettivo.

25. Clane Ralph, Fictions Inc. The Corporation in Postmodern Fiction, Film, and Popular Colture, Rutgens University Press, Londra -New Brunswick, 2014, p. 157.

26. Scarpa Tiziano, Kamikaze d'Occidente, cit., p. 217. 
possibilità di un ingaggio da parte di una rivista (cosa che permetterebbe al protagonista di lasciarsi alle spalle la vita da gigolo) non si concretizza e, contemporaneamente, il diario/cronaca della forma di vita occidentale si dimostra un successo (per la Cina), cosa che ribadisce la mancanza di alternative al mondo così com'è. In entrambi i casi è ancora una volta protagonista il denaro: l'ingaggio salta perché non viene firmato alcun contratto e il compenso da parte del ministero cinese si rivela un falso.

Scarpa descrive, dunque, la corsa di un uomo verso quella che si rivela in ultimo essere null'altro che una chimera; l'intera azione del protagonista volta alla riscossione del compenso si rivela essere un'illusione, quel denaro bramato sin dalle prime pagine - per quanto percepito come reale e tangibile - si manifesta in tutta la sua assenza. Il denaro è un elemento talmente legato alla speranza di emancipazione da rendere lecito tutto e piegare ogni cosa sotto il suo peso anche quando non c'è, anche quando il protagonista non riesca a guadagnare, a lavorare, a impiegarsi. In Kamikaze d'Occidente si assiste a una analisi dettagliata dello stato dell'economia italiana così come vissuta dal singolo, per cui neanche la caduta delle Torri gemelle o il G8 riescono a distogliere l'attenzione da una banconota da cinquanta euro; eppure, allo stesso tempo, c'è una rivolta contro la subordinazione dell'io, una reazione che si mantiene accesa anche nel momento in cui il soggetto perde i propri mezzi e diviene a sua volta una merce. Benché fallisca, in altre parole, il personaggio tenta instancabilmente di liberarsi dalle scintille del denaro.

"Non una speranza nel futuro, ma una speranza nel presente, non so se riesco a spiegarmi. Hanno creduto nel presente fino in fondo, hanno preso dai pochi attimi che gli restavano tutto quello che quegli attimi gli potevano dare, sapendo perfettamente che erano gli ultimi attimi, che non c'era niente da fare, solo viverli e basta".

"Non so se è molto attendibile come interpretazione, non credo che abbiano pensato la cosa in questi termini. Però hai commosso anche me guarda"27.

Lontano dal mondo a grandi cifre nordamericane, il romanzo di Scarpa si conforma - seppur non completamente - a una certa tendenza della narrativa italiana contemporanea a rappresentare esistenze precarie. Gli ambienti rappresentati dai romanzi e $\mathrm{i}$ racconti che tracciano questa tendenza sono tuttavia ben lontani da quello intellettuale e milanese di Kamikaze, il cui protagonista appartiene a una classe sociale che, sebbene in difficoltà, pubblica

27. Scarpa Tiziano, Kamikaze d'Occidente, cit., p. 194. 
e guadagna. Romanzi come La dismissione ${ }^{28} \mathrm{o}$ il più recente Ipotesi di una sconfitta ${ }^{29}$ si inseriscono a pieno titolo nella tradizione letteraria che da Volponi passa per Bianciardi e Ottieri e rappresentano il lavoro subordinandolo a una condizione di incertezza diffusa e ineluttabile ${ }^{30}$.

Il fallimento e la sottomissione al denaro, la consapevolezza della sconfitta e il nulla finale sembrerebbero i moti di un romanzo che non vuole concedere nulla al futuro, che non spera più: eppure Scarpa, grazie proprio all'uso che fa di uno strumento tipico della letteratura postmoderna - la metanarrazione: la spirale di uno scrittore che scrive un'opera su uno scrittore che sta scrivendo il testo che si legge - lancia un grido di allarme e comunica un moto di resistenza contro le derive del sistema economico:

Dopo un paio di mesi mi ha domandato: "Quanto vuoi per rifarlo?"

Quel "quanto vuoi per rifarlo?" è stato una svolta nella mia carriera. Mi ha fatto intravedere la possibilità di sbarcare il lunario senza stare sotto padrone.

"Guarda che sarà comunque un padrone. Un padrone diverso...", mi aveva avvertito lei, filosofica. Allora lavoravi per una fabbrica di riviste, con qualifica di operaio dell'alfabeto. Faticavo dieci ore al giorno alla pressa delle frasi, in redazione. Ho gettato il cuore oltre l'ostacolo, ho preso la rincorsa anch'io e ho fatto il salto: mi sono licenziato ${ }^{31}$.

Laura RINALDI

Université Paris Nanterre CRIX (Centre de Recherches Italiennes - Études Romanes)

Università di Torino

28. Rea Ermanno, La dismissione, Milano, Feltrinelli, 2002.

29. Falco Giorgio, Ipotesi di una sconfitta, Torino, Einaudi, 2017.

30. A questo proposito è centrale il dibattito che si è creato attorno a letteratura e azienda nella produzione italiana contemporanea. Importanti sono il numero 31/32 della rivista Narrativa (2010), intitolato Letteratura e azienda. Rappresentazioni letterarie dell'economia e del lavoro nell'Italia degli anni 2000, e CETERONI Alessandro, La letteratura aziendale. Gli scrittori che raccontano il precariato, le multinazionali e il nuovo mondo del lavoro, Milano, Calibano, 2018.

31. Scarpa Tiziano, Kamikaze d'Occidente, cit., p. 234. 